\title{
Windowless Silicon Drift Detectors
}

\author{
R. Anderhalt, A. Sandborg
}

EDAX Inc., 91 McKee Drive, Mahwah, NJ, 07430

Windowless EDS detectors have been used recently on the TEM -in one case as a liquid-nitrogen cooled, lithium-drifted silicon detector [1] and a silicon drift detector (SDD; [2]). The relatively modest, thermal electric cooling of the SDD is better suited to a modern windowless EDS application because the detector can be cooled in a few minutes and rapidly warmed when the TEM needs to be vented.

When the traditional window is not placed between the sample and the detector the improvement in transmission can be calculated and plotted. The windowless detector in figure 1 does include a light shield to reduce the effects of luminescence from the sample. The light shield is significantly thinner than the super ultra thin window (SUTW; Moxtek AP3.3) and does not require a support grid which further reduces the transmission of $\mathrm{X}$ rays. The most significant absorption edges shown in figure 1 are for carbon $(0.28 \mathrm{keV})$, for oxygen in the light shield $(0.53 \mathrm{keV})$, and a slight decrease from the aluminum coating used on both the light shield and SUTW $(1.56 \mathrm{keV})$.

The improvement in transmission is demonstrated in spectral comparisons between detectors with a SUTW and with no window (fig. 2). Spectral overlays are commonly auto-scaled to the highest peak in each spectrum but the improvement in transmission due to the lack of a support grid requires that each spectrum should be collected with the same beam current or normalized to the higher energy part of the spectrum where the $\mathrm{X}$ rays have sufficient energy to penetrate the support grid.

The windowless SDD is well suited to the TEM because of the high quality of the vacuum and the infrequency of venting the chamber. In the SEM the chamber is vented frequently and the SEM is commonly operated in low-vacuum conditions which makes it less suitable for windowless detectors.

\section{References}

[1] S. Isakozawa et al., Journal of Electron Microscopy (2010) 1093.

[2] H.S. von Harrach et al., Microsc. Microanal. 16 (Suppl 2) (2010) 1312. 


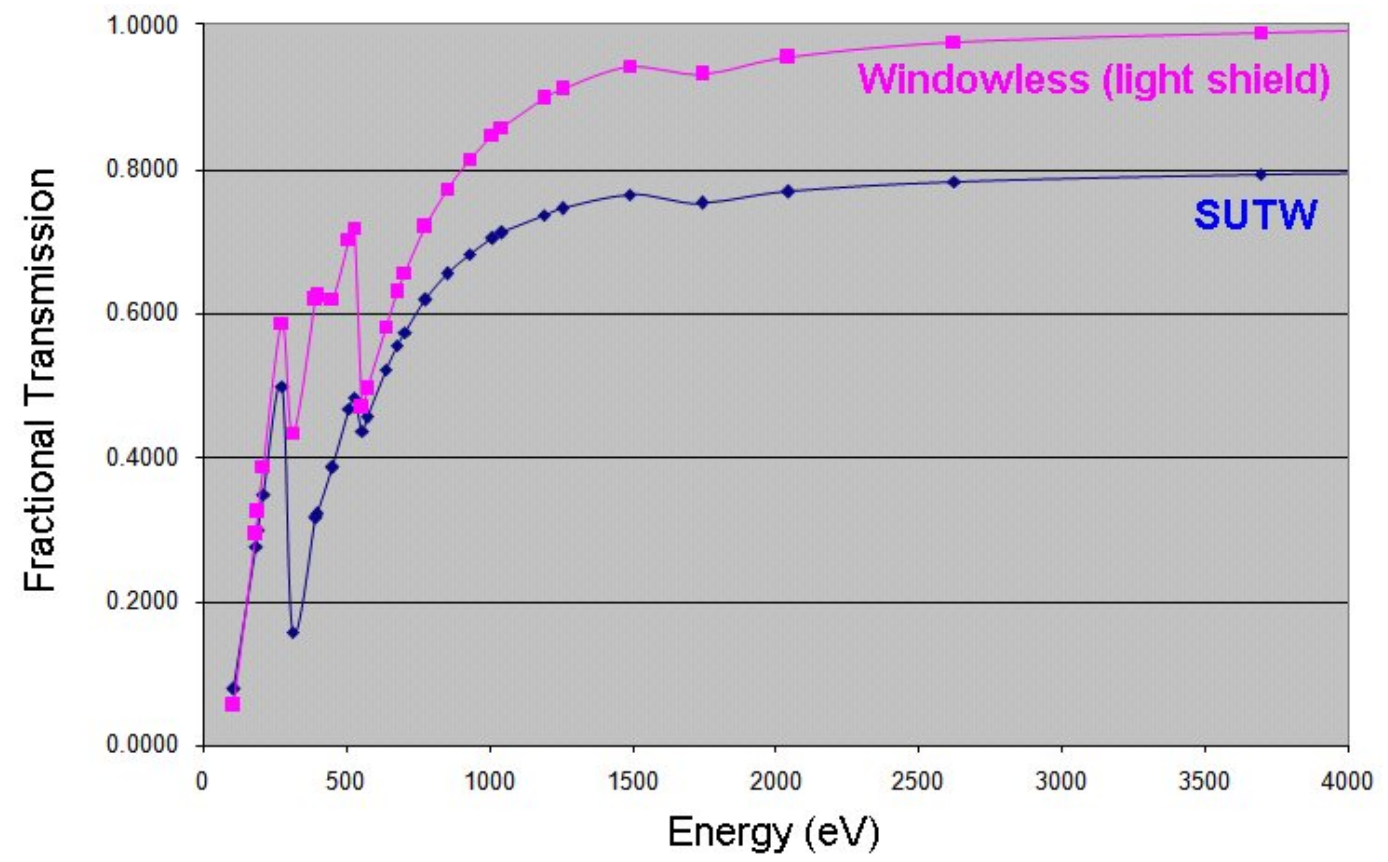

Fig. 1. Transmission plot versus energy for a super ultra thin window (SUTW; Moxtek AP 3.3) compared to a windowless detector with a light shield. Note that the SUTW detector requires a support grid which prevents a greater transmission in the higher energies plotted.

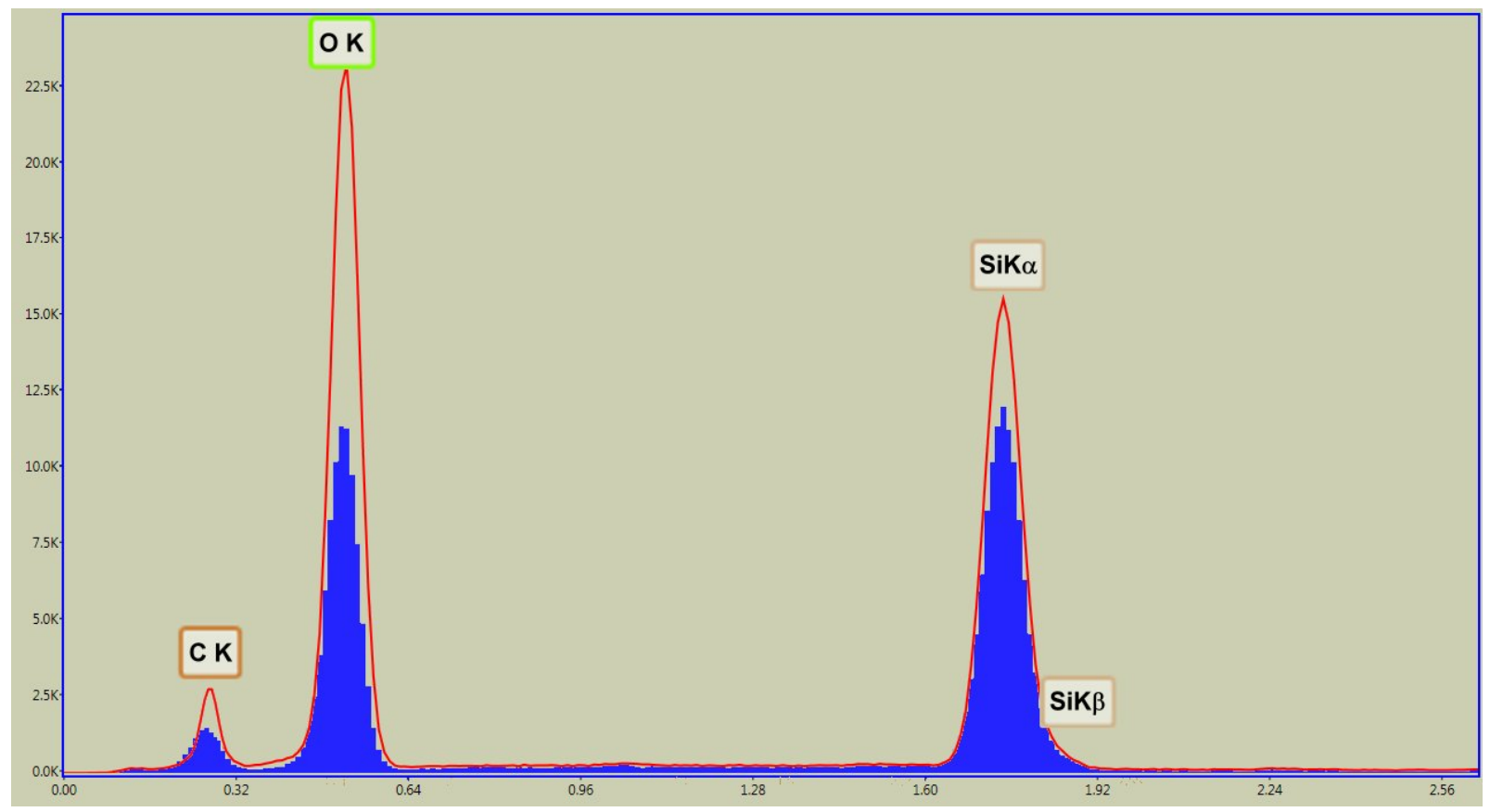

Fig. 2. Spectral overlay of $\mathrm{SiO}_{2}$ with a windowless detector (red outline) compared to the spectrum obtained with a detector fitted with a SUTW (shown in blue). These spectra have been normalized to each other at a higher energy range. The larger Si K $\alpha$ in the windowless spectrum is largely a result of the lack of a support grid. 